\title{
Geometry of almost Cliffordian manifolds: Nijenhuis tensor
}

\author{
Jaroslav Hrdina
}




\title{
GEOMETRY OF ALMOST CLIFFORDIAN MANIFOLDS: NIJENHUIS TENSOR
}

\author{
JAROSLAV HRDINA
}

\begin{abstract}
We generalize some classical results on Nijenhuis tensor for an almost Cliffordian manifold based on arbitrary Clifford algebra and suggest its relations with the integrability of the corresponding $G$-structure. We prove the set of properties for Nijenhus tensors with respect to arbitrary Clifford algebra.

2000 Mathematics Subject Classification: Primary 53C15; Secondary 53C10, 53C05

Keywords: Clifford algebra, Cliffordian manifold, Nijenhuis tensor, integrability, linear connections, $G$-structures
\end{abstract}

\section{Almost Cliffordian manifolds}

Let $\mathcal{O}=\ell \ell(s, t)$ be a Clifford algebra. If $M$ is an $\mathrm{km}$-dimensional manifold, where $k=2^{s+t}$ and $m \in \mathbb{N}$, then an almost Clifford manifold is given by a reduction of the structure group $\operatorname{GL}(\mathrm{km}, \mathbb{R})$ of the principal frame bundle of $M$ to

$$
\operatorname{GL}(m, \mathcal{O})=\{A \in \mathrm{GL}(\mathrm{km}, \mathbb{R}) \mid A I=I A, I \in \mathcal{O}\},
$$

where $\mathcal{O}$ is arbitrary Cliffford algebra. In other words, an almost Clifford manifold is a smooth manifold equipped by the set of anti commuting and commuting affinors $I_{i}, i=1, \ldots, t, I_{i}^{2}=-E$ and $J_{j}, j=1, \ldots, s, J_{j}^{2}=E$ such that the free associative unitary algebra generated by $\left\langle I_{i}, J_{j}, E\right\rangle$ is isomorphically equivalent to $\mathcal{O}$. In particular, on the elements of this reduced bundle, one can define affinors in the form $F_{1}, \ldots, F_{k}$ globally.

Definition 1. Let $M$ be a smooth manifold such that $\operatorname{dim}(M)=m$. Let $A$ be a smooth $\ell$-dimensional $(\ell<m)$ vector subbundle in $T^{*} M \otimes T M$ such that the identity affinor $E=\mathrm{id}_{T M}$ restricted to $T_{x} M$ belongs to $A_{x} M \subset T_{x}^{*} M \otimes T_{x} M$ at each point $x \in M$. We say that $M$ is equipped with an $\ell$-dimensional $A$-structure.

It is easy to see that an almost Clifford structure is not an $A$-structure because the affinors in the form $F_{0}, \ldots, F_{\ell} \in A$ have to be defined only locally.

The work supported by the Grant No. FSI-S-11-3. 
Definition 2. The $A$-structure, where $A$ is a Clifford algebra $\mathcal{O}$, is called an almost Cliffordian manifold.

In particular, the almost Clifford and almost Cliffordian structures are $G$-structures based on Clifford algebras. Two most important examples are an almost hypercomplex geometry and an almost quaternionic geometry, which are based on Clifford algebra $C \ell(0,2)$. Note that the geometric property of an almost hypercomplex structure reads that there is no nontrivial $G$-invariant subspace $\mathscr{D}$ in $\mathbb{\nabla} \otimes \wedge^{2} \mathbb{V}^{*}$ because the first prolongation $\mathfrak{g}^{(1)}$ of the Lie algebra $\mathfrak{g}$ vanishes. For an almost quaternionic structure, the situation is more complicated, because $\mathfrak{g}^{(1)}=\mathbb{V}^{*}$ and there is a class of these structures indexed by $\mathbb{V}$, see [1]. Note that for Cliffordian structures based on $\mathcal{C} \ell(0,3)$ is $\mathfrak{g}^{(1)}=\mathbb{V}^{*}$ and there is a class of these structures indexed by $\mathbb{V}$ too, see [2].

\section{NIJENHUIS TENSOR}

The Nijenhuis tensor plays an important role in the theory of integrability. As a classical concept, Nijenhuis introduced $N_{J} \in \wedge^{2} T^{*} M \otimes T M$ of an almost complex structure $J \in T^{*} M \otimes T M$. This tensor is an obstruction for an almost complex structure which distinguished it from the complex structure, i.e. their integrability. Recall that Nijenhuis tensor $N(P, Q) \in \wedge^{2} T^{*} M \otimes T M$ for a pair of tensors $P, Q \in$ $T^{*} M \otimes T M$ is given by the expression

$$
\begin{aligned}
N(P, Q)(X, Y)=[P X, Q Y] & -P[Q X, Y]-Q[X, P Y]+[Q X, P Y] \\
& -Q[P X, Y]-P[X, Q Y]+(P Q+Q P)[X, Y] .
\end{aligned}
$$

An almost quaternionic manifold $M$ is integrable if and only if the Nijenhuis tensors $N(I, I)$ and $N(J, J)$ vanish, where $I, J, I J \in T^{*} M \otimes T M$ is a quaternionic structure. Let us finally note that in [3], the author proved a similar fact for Clifford algebra $\mathcal{C} \ell(0,3)$.

If $P=Q$, then, by straightforward computing,

$$
N(P, P)(X, Y)=2\left([P X, P Y]-P[P X, Y]-P[X, P Y]+P^{2}[X, Y]\right)
$$

and if $P=E$, where $E$ is an identity, then

$$
N(E, Q)(X, Y)=0 .
$$

Following [6,7], we reformulate Nijenhuis tensor calculus with respect to arbitrary Clifford algebra. In those papers, a set of classical results for $\mathcal{\ell} \ell(0,2)$ and $\mathcal{C} \ell(2,0)$ was proved. In fact, most of the arguments work generally, but we have to formulate and prove them for any Clifford algebra. First of all, following papers [6,7], we recall the operation $\bar{\lambda}$

$$
(S \bar{\wedge} P)(X, Y):=S(P X, Y)+S(X, P Y)
$$


and

$$
(P \bar{\wedge} S)(X, Y):=P S(X, Y)
$$

where $S \in \otimes^{2} \mathbb{\nabla} \otimes \mathbb{V}^{*}$ and $N \in \mathbb{V} \otimes \mathbb{V}^{*}$. Now, one can easily check the following identities

$$
\begin{aligned}
N(L, Q P)+N(Q, L P)= & N(L, Q P) \bar{\wedge} P+L \bar{\wedge} N(Q, P) \\
& +Q \bar{\wedge} N(L, P) \\
(S \bar{\wedge} Q) \bar{\wedge} P-(S \bar{\wedge} P) \bar{\wedge} Q= & S \bar{\wedge} Q P-S \bar{\wedge} P Q \\
(L \bar{\wedge} S) \bar{\wedge} P= & L \bar{\wedge}(S \bar{\wedge} P)
\end{aligned}
$$

Lemma 1. Let $\mathcal{O}=\mathcal{C} \ell(s, t)$ be a Clifford algebra. If $F, G \in \mathcal{O}$ such that $F \neq G$, then the following identities hold:

$$
\begin{aligned}
N(F G, F) & =-\frac{1}{2} N(F, G) \bar{\wedge} F-\frac{1}{2} G \bar{\wedge} N(F, F)+\frac{1}{4} N(F, F) \bar{\wedge} G \\
0 & =N(F, G) \bar{\wedge} F+2 F \bar{\wedge} N(F, G)+G \bar{\wedge} N(F, F)+\frac{1}{2} N(F, F) \bar{\wedge} G \\
N(G, H) & =N(F, G) \bar{\wedge} G+G \bar{\wedge} N(F, G)+F \bar{\wedge} N(G, G) .
\end{aligned}
$$

Proof. Putting $L=Q=F, P=G$ in (3), we find

$$
N(F, F G)+N(F, F G)=N(F, F) \bar{\wedge} G+F \bar{\wedge} N(F, G)+F \bar{\wedge} N(F, G),
$$

that is,

$$
N(F G, F)=\frac{1}{2} N(F, F) \bar{\wedge} G+F \bar{\wedge} N(F, G) .
$$

Putting $L=G, P=Q=F$ in (3), we find

$$
N\left(G, F^{2}\right)+N(F, G F)=N(G, F) \bar{\wedge} F+G \bar{\wedge} N(F, F)+F \bar{\wedge} N(G, F),
$$

that is,

$$
N(F, G F)=N(G, F) \bar{\wedge} F+G \bar{\wedge} N(F, F)+F \bar{\wedge} N(G, F) .
$$

Adding (9) and (10) and dividing the sum by 2 , we find

$$
N(F G, F)=-\frac{1}{2} N(F, G) \bar{\wedge} F-\frac{1}{2} G \bar{\wedge} N(F, F)+\frac{1}{4} N(F, F) \bar{\wedge} G
$$

and subtracting (10) from (9), we find

$$
N(F, G) \bar{\wedge} F+2 F \bar{\wedge} N(F, G)+G \bar{\wedge} N(F, F)+\frac{1}{2} N(F, F) \bar{\wedge} G=0
$$

Finally, putting $L=P=G, Q=F$ in (3), we find

$$
N\left(F, G^{2}\right)+N(G, F G)=N(F, G) \bar{\wedge} G+F \bar{\wedge} N(G, G)+G \bar{\wedge} N(F, G),
$$

that is,

$$
N(G, H)=N(F, G) \bar{\wedge} G+G \bar{\wedge} N(F, G)+F \bar{\wedge} N(G, G) .
$$


Lemma 2. Let $\mathcal{O}=\mathcal{C} \ell(s, t)$ be a Clifford algebra. If $F, G \in \mathcal{O}$ such that $F \neq G$, then the following identity holds:

$$
\begin{aligned}
\epsilon_{1} N(F, F)-\epsilon_{2} N(G, G)+N(H, F) \bar{\wedge} G+G \bar{\wedge} N(H, F) \\
-N(G, H) \bar{\wedge} F+F \bar{\wedge} N(G, H)+2 H \bar{\wedge} N(F, G)=0,
\end{aligned}
$$

where $H=F G$ and $\epsilon_{i}=1$ for $K^{2}=-1$ and $\epsilon_{i}=-1$ for $K^{2}=1$.

Proof. Putting $L=F G, Q=F, P=G$ in (3), we find

$$
\begin{aligned}
N(F G, F G)-N(F, F G G)=N(F G, F) \bar{\wedge} G+F G \bar{\wedge} N(F, G) \\
+F \wedge N(F G, G),
\end{aligned}
$$

that is,

$$
\begin{aligned}
N(H, H)= & \epsilon N(F, F)+N(F G, F) \bar{\wedge} G+F G \bar{\wedge} N(F, G) \\
& +F \bar{\wedge} N(F G, G),
\end{aligned}
$$

where $\epsilon=1$ for $G^{2}=-1$ and $\epsilon=-1$ for $G^{2}=1$.

Putting $L=F G, Q=G, P=F$ in (3), we find

$$
\begin{aligned}
N(F G, G F)-N(G, F G F)= & N(F G, G) \bar{\wedge} F+F G \bar{\wedge} N(G, F) \\
& +G \bar{\wedge} N(F G, F),
\end{aligned}
$$

that is,

$$
N(H, H)=\epsilon N(G, G)-N(G, H) \bar{\wedge} F-H \bar{\wedge} N(F, G)-G \bar{\wedge} N(H, F),
$$

where $\epsilon=1$ for $G^{2}=-1$ and $\epsilon=-1$ for $G^{2}=1$. Thus, from (13) and (14), we find

$$
\begin{aligned}
N(H, H) & =\frac{1}{2}\{\epsilon N(F, F)+\epsilon N(G, G)+N(H, F) \bar{\wedge} G \\
& +G \bar{\wedge} N(H, F)-N(G, H) \bar{\wedge} F+F \bar{\wedge} N(G, H)+2 H \bar{\wedge} N(F, G)\}=0
\end{aligned}
$$

and

$$
\begin{aligned}
& \epsilon N(F, F)-\epsilon N(G, G)+N(H, F) \bar{\wedge} G \\
& +G \bar{\wedge} N(H, F)-N(G, H) \bar{\wedge} F+F \bar{\wedge} N(G, H)+2 H \bar{\wedge} N(F, G)=0,
\end{aligned}
$$

which completes the proof.

Lemma 3. Let $\mathcal{O}=\mathcal{\ell} \ell(s, t)$ be a Cliffod algebra. If $F \in \mathcal{O}$, then the following identity holds:

$$
N(F, F) \bar{\wedge} F=-2 F \bar{\wedge} N(F, F) .
$$

Proof. One can easily check that putting $L=P=Q=F$ gives $N\left(F, F^{2}\right)+$ $N\left(F, F^{2}\right)=N(F, F) \bar{\wedge} F+F \bar{\wedge} N(F, F)+F \bar{\wedge} N(F, F)$ 
Theorem 1. Let $\mathcal{O}$ be a Clifford algebra $\mathcal{\ell} \ell(s, t)$ and let $F, G \in \mathcal{O}$ such that $F \neq G$. If the Nijenhuis tensors $N(F, F)$ and $N(G, G)$ vanish, then $N(F G, F G)$ vanishes.

Proof. Since $N(F, F)=0$, we have from (9) $N(H, F)=F \bar{\wedge} N(F, G)$, and from (11),

$$
N(F, G) \bar{\wedge} F=-2 F \bar{\wedge} N(F, G) .
$$

Since $N(G, G)=0$, we have from (9), where we changed $F$ and $G$,

$$
N(F, G) \bar{\wedge} G=-2 G \bar{\wedge} N(F, G)
$$

and from (11), where we changed $F$ and $G$,

$$
N(F, G) \bar{\wedge} G=-2 G \bar{\wedge} N(F, G) .
$$

Now, if we substitute $N(F, F)=0, N(G, G)=0$, and (16) into (12), then the part containing $\epsilon_{i}$ vanishes and the proof is correct for any Clifford algebra, i. e., we find

$$
\begin{aligned}
(F \bar{\wedge} N(F, G)) \bar{\wedge} G+G \bar{\wedge}(F \bar{\wedge} N(F, G)) & \\
& -(G \bar{\wedge} N(F, G)) \bar{\wedge} F-F \bar{\wedge}(G \bar{\wedge} N(F, G))+2 H \bar{\wedge} N(F, G)=0,
\end{aligned}
$$

from which

$$
(F \bar{\wedge} N(F, G)) \bar{\wedge} G-(G \bar{\wedge} N(F, G)) \bar{\wedge} F=0,
$$

since

$$
G \bar{\wedge}(F \bar{\wedge} N(F, G))=-F \bar{\wedge}(G \bar{\wedge} N(F, G))=-H \bar{\wedge} N(F, G)
$$

by virtue of $G F=-F G=-H$. Now, using (5), (15) and (17), we find, from (18)

$$
\begin{aligned}
F \bar{\wedge}(N(F, G) \bar{\wedge} G)-G \bar{\wedge}(N(F, G) \bar{\wedge} F) & =0, \\
-2 F \bar{\wedge}(G \bar{\wedge} N(F, G))+2 G \bar{\wedge}(F \bar{\wedge} N(F, G)) & =0, \\
-4 F G \bar{\wedge} N(F, G) & =0,
\end{aligned}
$$

that is,

$$
H \bar{\wedge} N(F, G)=0 .
$$

Since $H^{2}=-1$, we have from (19) $N(F, G)=0$.

Corollary 1. Let $\mathcal{O}$ be a Clifford algebra $\mathcal{C} \ell(s, t)$. If the Nijenhuis tensors $N\left(I_{i}, I_{i}\right)$ vanish, where $I_{i}$ are the algebra generators of $\mathcal{O}$, then

$$
N\left(F_{i}, F_{j}\right)=0,
$$

where $F_{i}$ are vector space generators. 


\section{Classes of SUbordinAted CONNECTIONS}

Recall the concept of $A$-planar curves on $A$-structures equipped with the linear connection $\nabla$. For any tangent vector $X \in T_{x} M$, we shall write $A_{x}(X)$ for the vector subspace

$$
A_{x}(X)=\left\{F_{i}(X) \mid F_{i} \in A_{x} M\right\} \subset T_{x} M
$$

and call it the $A$-hull of the vector $X$. Similarly, A-hull of a vector field is a subbundle in $T M$ obtained pointwise. Let $M$ be a smooth manifold equipped with an $A$-structure and a linear connection $\nabla$. A smooth curve $c: \mathbb{R} \rightarrow M$ is said to be A-planar if

$$
\nabla_{\dot{c}} \dot{c} \in A(\dot{c}) .
$$

In [5], the authors proved a set of facts about the class of $\mathscr{D}$-connections. The theorems below, about Cliffordian structures, are proved in paper [5] and some examples of this concept can be found in papers $[2,4]$. The theorems about $\mathscr{D}$-connections can be found in [1].

Following [4,5], we have a set of results on Clifford and Cliffordian manifolds.

Corollary 2. Let $M$ be a smooth manifold equipped with a $G$-structure, where $G=\mathrm{GL}(n, \mathcal{O}), \mathcal{O}=\mathcal{C} \ell(s, t), s+t>1$, i. e. an almost Clifford manifold. Then the $G$-structure is of type 1 and there exists a unique $D$-connection.

One can see that an almost Cliffordian manifold $M$ is given as a $G$-structure provided that there is a reduction of the structure group of the principal frame bundle of $M$ to $G:=\operatorname{GL}(m, \mathcal{O}) \mathrm{GL}(1, \mathcal{O})=\operatorname{GL}(m, \mathcal{O}) \times \operatorname{GL}(1, \mathcal{O})$, the action of $G$ on $T_{x} M$ looks like $Q X q$, where $Q \in \mathrm{GL}(m, \mathcal{O}), q \in \mathrm{GL}(1, \mathcal{O})$, where the right action of $\operatorname{GL}(1, \mathcal{O})$ is blockwise. In this case the tensor fields in the form $F_{1}, \ldots, F_{k}$ can be defined only locally. It is easy to see that the Lie algebra $\mathfrak{g l}(m, \mathcal{O})$ of a Lie group $\mathrm{GL}(m, \mathcal{O})$ is of the form

$$
\mathfrak{g l}(m, \mathcal{O})=\left\{A \in \mathfrak{g l}(k m, \mathbb{R}) \mid A I_{i}=I_{i} A, A J_{j}=J_{j} A\right\}
$$

and the Lie algebra $\mathfrak{g}$ of a Lie group $\operatorname{GL}(m, \mathcal{O}) \operatorname{GL}(1, \mathcal{O})$ is of the form $\mathfrak{g}=\mathfrak{g l}(m, \mathcal{O}) \oplus$ $\mathfrak{g l}(1, \mathcal{O})$.

Let us note that the cases of $\ell \ell(s, t)$, where $s+t=2$, were studied in [6,7] and the case of $\mathcal{\ell} \ell(0,3)$ was studied in a detailed way in [2].

Corollary 3. Let $M$ be an almost Cliffordian manifold based on Clifford algebra $\mathcal{O}=\mathcal{C} \ell(s, t)$, where $\operatorname{dim}(M) \geq 2(s+t)$, i. e., a smooth manifold equipped with a $G$-structure, where $G=\mathrm{GL}(n, \mathcal{O}) \mathrm{GL}(1, \mathcal{O})$ or equivalently an A-structure, where $A=\mathcal{O}$. Then the class of $D$-connections preserves $A$ and shares the same A-planar curves, which are isomorphic to $\left(\mathbb{R}^{k m}\right)^{*}$. 


\section{CONCLUSION}

From the classical theory, the Nijenhuis tensor is a part of a torsion of any almost complex connection $\nabla$ and is $J$-antilinear in each argument,

$$
N_{J}(X, Y)=T_{\nabla}(X, Y)+J T_{\nabla}(J X, Y)+J T_{\nabla}(X, J Y)-T_{\nabla}(J X, J Y)
$$

and the connection $\nabla$ called minimal such that $N_{J}=4 T_{\nabla}$, i.e. the structure is integrable if and only if $N_{J}$ vanishes. Let $M$ be an almost quaternionic manifold or an almost quaternionic manifold of the second kind (paraquarernionic). In [6] and [7], the authors proved that the structure is integrable if and only if the structure tensor $T_{Q}=N(I, I)+N(J, J)+N(K, K)$ vanishes. In this case, there is the class of $\mathscr{D}$-connections without torsion.

A similar fact was proved for an almost Cliffordian manifold, where $\mathcal{C} \ell(0,3)$, see [3]. In this article, the autohors proved that the structure tensor which is locally generated by $F_{i}$ is given by

$$
T_{Q}=\sum_{i=1}^{6} N\left(F_{i}, F_{i}\right)+\sum_{i=1}^{6} N\left(F_{i}, F_{i}\right) \partial\left(\alpha_{a} \otimes F_{a}\right),
$$

where $\partial$ denotes Spencer's operator of alternation. The following step is to find a description of the structure preserving connection based on the Nijenhuis tensor for any Cliffordian manifold.

\section{REFERENCES}

[1] D. Alekseevsky and S. Marchiafava, "Quaternionic structures on a manifold and subordinated structures," Ann. Mat. Pura Appl., IV. Ser., vol. 171, pp. 205-273, 1996.

[2] I. Burdujan, "On almost Cliffordian manifolds," Ital. J. Pure Appl. Math., vol. 13, pp. 129-144, 2003.

[3] I. Burdujan, "Manifolds endowed with several complex structures," An. Ştiinţ. Univ. Al. I. Cuza Iaşi, Ser. Nouă, Mat., vol. 53, pp. 99-106, 2007.

[4] J. Hrdina and P. Vašik, "Generalized geodesics on almost cliffordian geometries," Balkan Journal of Geometry and Its Applications, vol. 17, no. 1, pp. 41-48, 2012.

[5] J. Hrdina and P. Vašik, "Geometry of almost Cliffordian manifolds: classes of subordinated connections," ArXiv e-prints, May 2012.

[6] K. Yano and M. Ako, "Integrability conditions for almost quaternion structures," Hokkaido Math. J., vol. 1, pp. 63-86, 1972.

[7] K. Yano and M. Ako, "Almost quaternion structures of the second kind and almost tangent structures," Kodai Math. Sem. Rep., 1973.

Author's address

Jaroslav Hrdina

Brno University of Technology Faculty of Mechanical Engineering, Institute of Mathematics, Brno, Czech Republic.

E-mail address: hrdina@fme.vutbr.cz 